\title{
Hedgehog signaling pathway and its targets for treatment in basal cell carcinoma
}

\author{
This article was published in the following Dove Press journal: \\ Journal of Experimental Pharmacology \\ 14 December 2012 \\ Number of times this article has been viewed
}

\author{
Danilo Cucchil,* \\ Maria Anna Occhione ${ }^{2, *}$ \\ Alberto Gulino ${ }^{2,3}$ \\ Enrico De Smaele' \\ 'Department of Experimental \\ Medicine, ${ }^{2}$ Department of Molecular \\ Medicine, Sapienza University of Rome, \\ Rome, ${ }^{3}$ Center of Life NanoScience \\ @ La Sapienza, Istituto Italiano di \\ Tecnologia, Rome, Italy \\ *These authors contributed equally \\ to this work
}

Correspondence: Enrico De Smaele Department of Experimental Medicine, Sapienza University of Rome, Viale Regina Elena 324, 0016I Rome, Italy

$\mathrm{Tel}+3906492455659$

$\mathrm{Fax}+390649255660$

Email enrico.desmaele@uniromal.it
Abstract: Basal cell carcinoma (BCC) of the skin is the most common type of cancer and accounts for up to $40 \%$ of all cancers in the US, with a growing incidence rate over recent decades in all developed countries. Surgery is curative for most patients, although it leaves unaesthetic scars, but those that develop locally advanced or metastatic BCC require different therapeutic approaches. Furthermore, patients with BCC present a high risk of developing additional tumors. The increasing economic burden and the morbidity of $\mathrm{BCC}$ render primary interest in the development of targeted treatments for this disease. Among the molecular signals involved in the development of BCC, the critical role of the morphogenetic Hedgehog (Hh) pathway has become evident. This pathway is found altered and activated in almost all BCCs, both sporadic and inherited. Given the centrality of the Hh pathway in the pathophysiology of BCC, the primary efforts to identify molecular targets for the topical or systemic treatment of this cancer have focused on the Hh components. Several Hh inhibitors have been so far identified - from the first identified natural cyclopamine to the recently Food and Drug Administration-approved synthetic vismodegib - most of which target the Hh receptor Smoothened (either its function or its translocation to the primary cilium). Other molecules await further characterization (bisamide compounds), while drugs currently approved for other diseases such as itraconazole (an antimicotic agent) and vitamin D3 have been tested on BCC with encouraging results. The outcomes of the numerous ongoing clinical trials are expected to expand the field in the very near future. Further research is needed to obtain drugs targeting downstream components of the Hh pathway (eg, Gli) or to exploit combinatorial therapies (eg, with phosphatidylinositol 3-kinase inhibitors or retinoids) in order to overcome potential drug resistance.

Keywords: BCC, Hedgehog, vismodegib, Smo inhibitors, Gli antagonists, retinoids, itraconazole, vitamin D3

\section{Introduction}

Basal cell carcinoma (BCC) of the skin is the most common type of cancer and accounts for up to $40 \%$ of all cancers in the US. ${ }^{1}$ BCC, together with squamous cell carcinoma, is grouped into the so-called nonmelanoma skin cancers which present cells with keratinocytic morphology. ${ }^{2}$

$\mathrm{BCC}$ is characterized by cells that morphologically resemble the undifferentiated cells that constitute the basal layer of the epidermis or hair follicle, but may present different growth patterns (infiltrating, superficial, nodular, sclerosing, and fibroepithelial), indicating that the cell of origin may be a stem or progenitor cell.,

BCC tumors are generally curable and thus frequently not reported in cancer registries, making it difficult to estimate the real incidence of the disease. A report 
estimated BCC occurrence in the US of more than 2 million cases in 2006, with some patients having multiple diagnoses. ${ }^{5}$ In $95 \%$ of cases, the diagnosis is made in 40-79 year olds mainly of Caucasian origin, with a $30 \%$ higher incidence in men than in women. ${ }^{6}$

Exposure to sunlight (ultraviolet rays) is the most important risk factor (in most cases the lesion is in a region of the body exposed to the sun); other well known risk factors are exposure to ionizing radiations, arsenic, and immunosuppressive conditions as well as genetic syndromes such as the basal cell nevus syndrome (BCNS) or Gorlin syndrome. ${ }^{3,6}$

Despite its very high frequency, this tumor rarely leads to death, partly because of the remarkable progress made in the field of diagnosis and treatment, but also for the fact that it rarely metastasizes. However, since BCC often infiltrates the skin locally and aggressively and involves disfiguring surgical resection, it is considered a malignant tumor. It is important to observe that metastatic patients, although very rare $(0.003 \%-0.5 \%$ of cases $)$, often present a poor prognosis with mean survival ranging from 8 months to 3.6 years. ${ }^{7}$

A recent systematic review of the published data suggests that the incidence rate of $\mathrm{BCC}$ has grown in recent decades in developed countries, probably because of the now widespread practice of sunbathing. ${ }^{8}$ The increasing relevance of the economic burden and the morbidity of BCC render primary interest in a better understanding of the molecular biology of BCC and the development of targeted treatments for this disease. ${ }^{9}$

\section{Current therapeutic approaches}

Surgery is still the elective choice for most BCCs, especially the early-stage and low-risk ones, which can be removed by surgical excision, electrodessication, curettage (tissue destruction by electric current and removal by scraping with a curette), or cryosurgery (tissue destruction by freezing). In the presence of specific risk factors, Mohs micrographic surgery is the elective treatment. ${ }^{10}$ Surgery is curative for most patients, although it leaves unaesthetic scars, but those that develop locally advanced or metastatic BCC require different therapeutic approaches. ${ }^{10}$

Furthermore, patients with BCC present a high risk of developing additional tumors (up to $44 \%$ incidence within 3 years from the first tumor), ${ }^{11}$ and some sort of preventive treatment on these patients may avoid the need for performing surgical resection again (which often causes disfigurement).

Until last year, the most common nonsurgical therapies for BCC involved radiotherapy, photodynamic therapy, or the topical administration of imiquimod and 5-fluorouracil, although the cure rates were low. ${ }^{10,12}$ Treatment of BCC with other agents, such as tazarotene, glycoalkaloid (BEC5) cream, cidofovir, and calcium dobesilate have also been reported, ${ }^{13}$ although many of them did not look really promising, prompting the search for more targeted and preventive therapies.

\section{Development of new BCC anticancer drugs: targeting the Hedgehog (Hh) pathway}

Among the molecular signals involved in the development of $\mathrm{BCC}$, the critical role of the morphogenetic Hh pathway has become evident. ${ }^{14,15}$ This pathway is found altered and activated in almost all BCCs, both sporadic and inherited, ${ }^{16,17}$ as well as in most tumor stem cells and in other tumors such as medulloblastoma and rhabdomyosarcoma. ${ }^{17-19}$

The Hh pathway is a key regulator of embryonic patterning and cell fate determination. ${ }^{20}$ It plays a critical role in the development of various tissues in mammals as a morphogenetic, mitogen and differentiation factor. ${ }^{21-26}$

The Hh pathway (Figure 1) is activated by a family of secreted ligands (Sonic, Desert, and Indian Hh). These ligands are synthesized as pro-proteins, which undergo several posttranslational modifications: autocatalytic cleavage to generate the active n-terminal fragment followed by linking to a cholesterol molecule and to a palmitic acid. ${ }^{27,28}$ These modifications are essential for $\mathrm{Hh}$ proteins to cross the membrane bilayers and to interact physically with lipoproteins, allowing diffusion of the ligand towards the nearby or distant target cells where Hh interacts with the transmembrane receptor Patched (Ptch). ${ }^{27,28}$ This bond prevents the inhibition mediated by Ptch on the coreceptor Smoothened (Smo), allowing Smo derepression and translocation in the primary cilium and leading to the downstream activation of the Gli family of transcription factors (Gli1-3). ${ }^{14,15,29}$

Recent studies suggest that Smo signaling activity can be modulated positively by natural and synthetic small molecules, including oxysterols (oxidized forms of cholesterol), ${ }^{30}$ and Ptch regulates Smo by pumping away oxysterols from Smo. ${ }^{31}$ Indeed, Ptch receptor contains a sterol sensing domain that has been linked to the regulation of the vesicular trafficking of Smo. ${ }^{32}$

Specific oxysterols can drive differentiation of osteogenic cells in culture and in animals. ${ }^{33,34}$ The most potent activator so far identified is 20(S)-hydroxycholesterol, which binds Smo and leads to its accumulation in the primary cilium. ${ }^{35,36}$ 


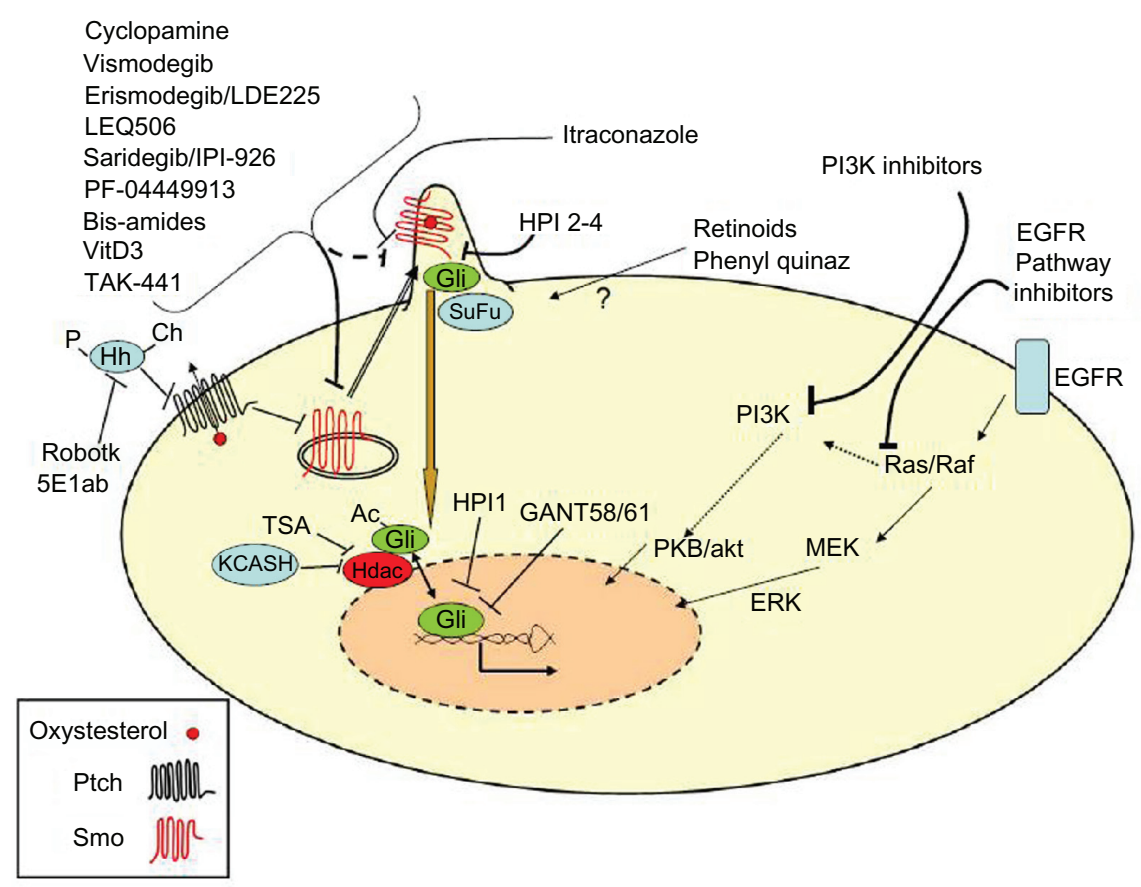

Figure I The Hh pathway critical components which could be targeted by potential antibasal cell carcinoma drugs.

Notes: The mechanism of action of each single drug is explained in the text. The question mark indicates a so far undetermined mechanism. The EGFR and PI3K pathways are also sketched, given their potential contribution to Hh pathway tumorigenic activity.

Abbreviations: Ac, acetyl group; Ch, cholesterol; EGFR, epidermal growth factor receptor; ERK, extracellular signal-related kinase; GANT, Gli antagonist; Hdac, histone deacetylase; Hh, Hedgehog; HPI, Hedgehog pathway inhibitor; KCASH, KCTD (Potassium Channel Tetramerization Domain) containing cullin3 adaptor suppressor of hedgehog; MEK, mitogen-activated protein kinase kinase; P, palmitate; PI3K, phosphatidylinositol 3-kinase; PKB/akt, protein kinase B; Ptch, Patched; Quinaz, quinazolines; Robotk, robotnikinin; Smo, Smoothened; SuFu, suppressor of fused; TSA, trichostatin A; VitD3, Vitamine D3.

Once activated, Gli transcription factors translocate into the nucleus and promote the expression of target genes. Recently, it has been demonstrated that Gli transcriptional activity and nuclear localization are also modulated negatively by acetylation. In fact, deacetylation by histone deacetylases is required for full transcriptional activity. ${ }^{37}$ Gli proteins promote the expression of genes involved in the control of the most important cell functions such as proliferation, differentiation, and survival. Dysregulated Hh activity is for this reason frequently implicated in cancerogenesis. ${ }^{38}$

The first hint of Hh involvement in BCC came from the analysis of the inherited forms of $\mathrm{BCC}$, associated with BCNS, an autosomal dominant disorder characterized by skeletal abnormalities, endocrine disorders, and predisposition to the development of BCC and medulloblastoma, ${ }^{39}$ which was found to be caused by a germline mutation of the $\mathrm{Hh}$ receptor Ptch1..$^{40,41}$

Like the hereditary form of $\mathrm{BCC}$, the most common genetic alterations found in sporadic $\mathrm{BCC}$ are mutations of the receptor Ptch1 (inactivating mutations or loss of heterozygosity), ${ }^{42-44}$ which prevent its inhibition on Smo receptor - allowing the activation of downstream signals even in the absence of ligands. ${ }^{42}$ Less frequent genetic alterations are gain of function mutations on Smo or mutations of the $\mathrm{Hh}$ inhibitor suppressor of fused (SuFu) ${ }^{43,45-47}$ Similarly, mouse models overexpressing Sonic Hh (Shh) ligand in the skin present BCC formation, ${ }^{48}$ although human BCCs generally do not present this alteration.

Given the centrality of the Hh pathway in the pathophysiology of BCC, the primary efforts to identify molecular targets for the topical or systemic treatment of this cancer have focused on the Hh components (Figure 1; Table 1).

\section{Development of Smo inhibitors Cyclopamine}

The first Hh inhibitor that has been identified is cyclopamine, a natural molecule found in Veratrum californicum (corn lilies) and recognized as a teratogen in cattle since the second half of the 1900s. ${ }^{49}$ The suppression of the Hh pathway through inhibition of Smo was later identified as the mechanism of the teratogenic malformation induced by cyclopamine..$^{50,51}$

The feasibility of cyclopamine use in therapy was suggested by its Hh inhibition on tumor cells. ${ }^{52,53}$ Cyclopamine (and its more effective derivative KAAD-cyclopamine) was successfully used topically on BCC, although with an inconvenient treatment schedule, ${ }^{54}$ verifying the rationale for the use of this class of molecules for cancer treatment. 
Table I List of the anti-Hedgehog drugs currently undergoing clinical trials

\begin{tabular}{|c|c|c|c|c|}
\hline Drug & Target & Cancer type & Phase & Clinicaltrials.gov ID \\
\hline \multirow[t]{13}{*}{ Vismodegib } & Smo & BCNS & II & NCT00957229 \\
\hline & & Advanced $/ \mathrm{mBCC}$ & II & NCT0I367665 \\
\hline & & $\mathrm{BCC}$ & II & NCTOI201915 \\
\hline & & Advanced BCC & II & NCT008334I7 \\
\hline & & Advanced $/ \mathrm{mBCC}$ & II & NCTOII 60250 \\
\hline & & Solid tumors & II & NCT00959647 \\
\hline & & Colorectal & II & NCT006366I0 \\
\hline & & Ovarian & II & NCT0073966I \\
\hline & & $\mathrm{BCC}$ & II & NCT0I54358I \\
\hline & & Medulloblastoma & I & NCT0I60II84 \\
\hline & & Pancreatic & 1 & NCT0I537I07 \\
\hline & & Medulloblastoma & II & NCT0I2393I6 \\
\hline & & Medulloblastoma & II & NCT00939484 \\
\hline \multirow[t]{6}{*}{ LDE225 } & Smo & Solid tumors & 1 & NCT00880308 \\
\hline & & & & NCT0I20883I \\
\hline & & BCNS & II & NCT0096I896 \\
\hline & & $\mathrm{BCC}$ & II & NCT0I0330I9 \\
\hline & & Advanced $/ \mathrm{mBCC}$ & II & NCT0I 327053 \\
\hline & & BCNS & II & NCT0I350II5 \\
\hline LEQ506 & Smo & Solid tumors & 1 & NCT0II 06508 \\
\hline \multirow[t]{2}{*}{ IPI-926 } & Smo & Solid tumors & 1 & NCT0076I696 \\
\hline & & Pancreatic & $\mathrm{lb} / \mathrm{II}$ & NCT0II30I42 \\
\hline TAK-44I & Smo & Solid tumors & 1 & NCTOI 204073 \\
\hline \multirow[t]{3}{*}{ PF-044499I3 } & Smo & Hematologic & I & NCT00953758 \\
\hline & & & & NCT0I546038 \\
\hline & & Solid tumors & 1 & NCT0I 286467 \\
\hline Itraconazole & Smo & $\mathrm{BCC}$ & II & NCT0II 08094 \\
\hline Vitamin D3 & Smo & $\mathrm{BCC}$ & III & NCTOI 358045 \\
\hline Acitretin & $?$ & NMSC & II & NCT00020956 \\
\hline Tretinoin & $?$ & NMSC & III & NCT0000763I \\
\hline \multirow[t]{2}{*}{ Tazarotene } & $?$ & $\mathrm{BCC} / \mathrm{BCNS}$ & II & NCT00489086 \\
\hline & & & II & NCT00783965 \\
\hline
\end{tabular}

Abbreviations: BCC, basal cell carcinoma; BCNS, basal cell nevus syndrome; mBCC, metastatic BCC; NMSC, nonmelanoma skin cancers; Smo, smoothened; ?, currently undefined target.

The availability of more potent and bioavailable derivatives (see below) has currently halted cyclopamine clinical development.

\section{Vismodegib (GDC-0449)}

Vismodegib (GDC-0449) belongs to the second class of Hh inhibitors, molecules capable of binding the target with higher affinity. ${ }^{55,56}$ Vismodegib was identified in a high-throughput screening of molecules optimized by means of targeted chemical modifications. ${ }^{57}$ In 2009 , Von Hoff et al published the promising results of a Phase I clinical trial of patients with locally advanced or metastatic BCC: of the 33 enrolled patients, 18 showed response to treatment (two complete and the other 16 partial) and the remaining 15 showed stable disease $(n=11)$ or progression $(n=4) .{ }^{58}$ Following this trial, a $150 \mathrm{mg} /$ day trial with 104 patients showed a 30\% response rate per metastatic $\mathrm{BCC}$ and a $43 \%$ rate per locally advanced BCC. Adverse events have proved tolerable. ${ }^{59}$
Given these results, vismodegib was approved by the Food and Drug Administration (FDA) in January 2012 for the treatment of locally advanced or metastatic BCC, ie, for those patients for whom it is impossible to resort to surgery or radiotherapy. ${ }^{56,60}$

Later on during 2012, results from one more clinical trial were published: Tang et al administered the drug to patients with BCNS and demonstrated its effectiveness in reducing preexisting lesions (mean $-65 \%$ versus $-11 \%$ placebo) and inhibiting new lesion formation (average two versus 29 per year). ${ }^{61}$ Histological samples taken from patients who received the treatment for at least 1 month showed a reduction of $90 \%$ in Hh activity (measured by the levels of Gli1).

Although the efficacy of the therapy was striking and led to quick acceptance, given its low toxicity, questions still remain that prompt further research in the field:

- First of all, despite these positive results, trials have indicated that after discontinuation of therapy a 
resumption of growth may occur. ${ }^{61}$ One hypothesis is that treatment is not able to kill a fraction of the cells that remain quiescent, and when treatment is suspended this population gives rise to full blown BCC.

- Second, a phenomenon observed during the use of vismodegib and potentially other inhibitors of Smo is drug resistance: it has been observed in medulloblastoma patients who have already undergone conventional treatments, so it may not occur with the same frequency in BCC patients - especially those who can be considered chemonaïve. ${ }^{62}$ A potential mechanism for this resistance is the acquisition of a Smo mutation (D473H), which does not allow vismodegib binding but retains its ability to activate the pathway. ${ }^{63}$

\section{Other Smo inhibitors}

In the last 10 years, several companies have invested in the search for good inhibitors of $\mathrm{Hh}$, with variable results. Since the inhibition of Smo is the most well-known mechanism for interfering with the Hh pathway (as shown by the success of cyclopamine and vismodegib), several molecules have been developed for this purpose. ${ }^{55}$

\section{Cur-6I4I4}

One of the first attempts was made by researchers at Curis Inc (Lexington, MA) with Cur-61414, which - although successful in a mouse model of $\mathrm{BCC}$ - failed to provide positive results in human $\mathrm{BCC}$, probably because of inadequate penetration in the skin. ${ }^{64,65}$

\section{NVP-LDE225, erismodegib}

A more promising inhibitor is LDE225 (NVP-LDE225, erismodegib) a new and specific inhibitor of Smo developed by Novartis (Basel, Switzerland), usable both locally and systemically (orally). ${ }^{66}$

Skvara et al, after having shown that topical LDE225 is able to reverse the formation of basaloid tumors in skin punches taken from mice Ptch $1^{+-}$, enrolled eight patients suffering from BCNS: a total of 27 lesions were treated twice a day with cream LDE225 $0.75 \%$ for 4 weeks. ${ }^{67}$ Histopathology showed a reduction in Ki-67 staining and a reduction in nuclear Gli1 reactivity in the lesions treated compared to controls; accordingly, analysis by quantitative polymerase chain reaction showed a reduction in the levels of Gli1, Gli2, and Ptch1 messenger ribonucleic acid from -2 to -16 times in the treated lesions. Of the 13 lesions treated with the drug, twelve showed a response - three complete, understood as the disappearance of the lesions and nine partial, intended as a reduction of the lesions. None of the lesions showed evidence of complete histological clearance - Skvara et al suggest the existence of a subpopulation of resistant cells (cancer stem cells?), which may lead to tumor regrowth. ${ }^{4,67}$ Although other conclusive data have not yet been published, Novartis has suspended the development of the topical LDE225 cream since the clinical studies did not demonstrate tumor clearance rates sufficient enough to support further clinical investigation. ${ }^{68}$

On the contrary, there are currently several ongoing clinical trials to test efficacy of oral administration of LDE225 in patients:

- a Phase I study of oral LDE225 in patients with advanced solid tumors (BCC, medulloblastoma, and others) (NCT00880308);

- a Phase II, randomized, double-blind study of efficacy and safety of two dose levels of LDE225 in patients with locally advanced or metastatic BCC (NCT01327053); and

- a Phase II trial evaluating the efficacy, safety, and pharmacokinetics of oral LDE225 in the treatment of adult patients with BCNS (NCT01350115).

A recently published paper indicates that NVP-LDE225 monotherapy in chemonaïve tumors had little effect on tumor growth. ${ }^{69}$ Notably, however, NVP-LDE225 treatment was highly effective in preventing the recurrence of residual tumors following chemotherapy. ${ }^{69}$

\section{LEQ506}

Another Novartis Smo inhibitor is LEQ506, ${ }^{70,71}$ which is currently undergoing a Phase I clinical trial on advanced solid tumors: recurrent or refractory medulloblastoma or locally advanced or metastatic BCC (NCT01106508), for which results are not currently available.

\section{IPI-926 (saridegib)}

Infinity Pharmaceuticals (Cambridge, MA) has developed a new molecule using the cyclopamine structure as starting material: making a series of chemical modifications to the ring system of the molecule has generated IPI-926 (saridegib) characterized by a greater chemical stability, solubility, potency, selectivity, and bioavailability compared to the natural compound. ${ }^{72-74}$

Recently, Lee et al demonstrated that saridegib lengthens the life of an Hh-dependent mouse model of medulloblastoma. ${ }^{75}$ In this study, $\mathrm{Ptch}^{\mathrm{C} / \mathrm{C}}$ animals were treated for 6 weeks, with $100 \%$ survival in saridegib-treated mice versus $0 \%$ of controls and a reduction in clinical symptoms 
in treated animals. ${ }^{75}$ Lee et al also suggested that saridegib reduces tumor-initiating capacity since they observed reduced tumor incidence, slower growth, and spontaneous tumor regression in allografts generated from previously treated autochthonous medulloblastomas compared with those from untreated donors. Lee et al did not observe the appearance of mutations at the level of Smo nor other resistance mechanisms such as amplification of Gli2 (actually, saridegib is active on cells transfected with a Smo mutant resistant to vismodegib, D473H). Despite this, a recovery in tumor growth accompanied by a progressive increase in the levels of Gli1 was observed after the 6 weeks of treatment. ${ }^{75}$

The hypothesis presented is that saridegib would seem to interfere with the activity of adenosine triphosphate binding cassette transporters (ABC) such as P-glycoprotein. $\mathrm{ABC}$ transporters are membrane proteins that use adenosine triphosphate for a conformational change that transport a variety of substrates across the cell membranes to the exterior and are also known for their capability as drug transporters out of the cells, making it difficult for the drug to reach an effective concentration in the cells. ${ }^{76,77}$ In fact, an increase of P-glycoprotein expression was observed in saridegib-treated tumors while administration of verapamil (an inhibitor of P-glycoprotein) partially restored the reduction in Gli1 levels. ${ }^{75}$

The effect of saridegib on P-glycoprotein casts some doubt on the feasibility of combined therapies, given the fact that stronger extracellular transport means reduced drug efficacy, and several authors are considering $\mathrm{ABC}$ transporters as potential targets for therapy. ${ }^{78}$ Interestingly, on the contrary, Hhantag691 administration has been linked to P-glycoprotein inhibition, ${ }^{79}$ while the activity of vismodegib on these molecules has been evaluated as modest. ${ }^{80}$

Oral IPI-926 is currently undergoing clinical experimentation: a Phase I study on IPI-926 has been completed (NCT00761696) and preliminary results indicate that IPI-926 was well tolerated and resulted in clinical activity in patients with BCC. ${ }^{81}$ Further studies (a Phase Ib/II study) are ongoing to address the potential of a combination with gemcitabine in patients with more aggressive cancers (ie, metastatic pancreatic cancer) (NCT01130142).

\section{Pfizer PF-04449913}

Pfizer, Inc (New York, NY) has developed its own Hh inhibitor, PF-04449913, an orally bioavailable Smo antagonist and benzimidazole derivative. ${ }^{82}$ This molecule is currently being tested in Phase I clinical trials in hematologic malignancies (NCT00953758, NCT01546038) and selected advanced, metastatic solid tumors (NCT01286467) both as a single agent or in combination with standard chemotherapy agents. Recently presented preliminary data highlight the good tolerability of the drug, and early signs of efficacy have been observed in all hematologic diseases studied. ${ }^{83}$ Treated patients presented downregulation of the antiapoptotic protein B-cell lymphoma-2 $(\mathrm{Bcl} 2)$ and the transporter protein $\mathrm{ABCA} 2 .{ }^{84}$ Although not yet tested on BCC, the efficacy on BCC could be assessed using a topical formulation since the current formulation is in tablets.

\section{TAK-44I}

Millennium Pharmaceuticals, Inc (Cambridge, MA) which is part of Takeda Pharmaceutical Company Ltd (Osaka, Japan) has launched a clinical trial on the oral administration of TAK-441, its Hh signaling pathway inhibitor (NCT01204073). This study involves adult patients with advanced nonhematological malignancies, but at the time of this review results were not yet available.

\section{Bisamides}

Dijkgraaf et al analyzed tumors with the Smo D473H mutation, resistant to vismodegib, and demonstrated an ability to overcome this resistance using a new type of inhibitor belonging to the class of bisamides. ${ }^{85}$ Confirming the validity of this approach, a research group from AstraZeneca (London, UK) recently identified another bisamide with potent Smoinhibiting activity and good pharmacokinetics in mice. ${ }^{86}$ Both molecules, although promising, need further characterization in vitro and/or in vivo and to be validated in clinical trials.

\section{Itraconazole}

After a screening for inhibitors of Hh using about 2400 molecules previously tested for toxicity in humans or approved by the FDA, Kim et al identified itraconazole as a potent inhibitor of $\mathrm{Hh} .{ }^{87}$ Itraconazole is an antifungal medication commonly used in clinical practice, even for long periods of time; it inhibits the enzyme 14- $\alpha$ lanosterol demethylase, crucial for the biosynthesis of ergosterol in fungi and cholesterol in mammals. ${ }^{9}$

Itraconazole gave positive results both in xenograft models of medulloblastoma and in the reduction of endogenous BCCs in murine models. ${ }^{87}$ According to Kim et al, the inhibitory effect on $\mathrm{Hh}$ by itraconazole was not mediated by its action on the synthesis of sterols, since the drug acted downstream of Ptch; on the contrary, itraconazole prevented accumulation of Smo in the primary cilium. Furthermore, the drug was able to synergize with cyclopamine, suggesting that the two drugs recognized two different binding sites on Smo. ${ }^{87}$ 
An exploratory Phase II proof of concept clinical trial with itraconazole on BCC patients has been performed by Stanford University (NCT01108094) with positive results, although additional studies with larger numbers of patients are needed to validate these findings. ${ }^{88}$

An important aspect of itraconazole is that it has been shown to also inhibit angiogenesis through inhibition of vascular endothelial growth factor receptor trafficking and downstream signaling. ${ }^{89}$ Therefore, its actions may be exerted simultaneously on both of these important therapeutic targets.

Following the discovery of itraconazole's potential for Hh inhibition, an interesting attempt has been performed to search for analogs of itraconazole with greater potency: 25 itraconazole side chain analogs were synthesized obtaining molecules with greater inhibitory activity. ${ }^{90}$ This work also helped identify the distinct regions critical for anti-Hh and antivascular endothelial growth factor receptor activity. Further preclinical and clinical work is now needed to validate these new compounds.

\section{Phenyl quinazolines}

Another promising class of Hh inhibitors is a small family of phenyl quinazolinone ureas, which has been reported as potent modulators of $\mathrm{Hh}$ protein function. Preliminary structure-activity relationship studies of the urea substituent led to a nanomolar Hh antagonist (compound $7 \mathrm{~d}$ ), which will need further testing for specificity, toxicity, and bioavalaibility. ${ }^{91}$

\section{Vitamin D3 (calcitriol)}

The literature provides wide evidence for the antitumoral activity of vitamin D3, showing its capabilities of blocking the cell cycle, inducing differentiation and apoptosis, modulating inflammation, and inhibiting neoangiogenesis. ${ }^{92,93}$

Vitamin D3 was shown to act both by binding to its nuclear receptor (VDR) and activating transcription (genomic effects), and by nongenomic effects, such as increasing the influx of calcium $\left(\mathrm{Ca}^{+}\right) .{ }^{93} \mathrm{~A}$ relationship between vitamin D3 and the Hh pathway came about by the observation of a Ptch-dependent secretion of provitamin D3 and by Smo inhibition by provitamin D3 or vitamin D3 itself. ${ }^{94,95}$ Furthermore, mice lacking VDR present higher levels of $\mathrm{Hh}$ signaling components in the epidermis and epidermal portion of the hair follicles. ${ }^{96}$

The relevance of vitamin D3 and VDR in BCC tumorigenesis has been suggested by Kamradt et al, while the involvement of $\mathrm{Hh}$ in this mechanism was highlighted by an analysis of BCC formation in VDR null mice. ${ }^{96,97}$
The relevance of exploiting vitamin D3 in BCC chemotherapy has been demonstrated on mice overexpressing the Hh pathway in the skin, in which vitamin D3 leads to VDR activation and induces Hh repression, inhibiting BCC proliferation and inducing differentiation. ${ }^{98}$

Since vitamin D3 does not seem to affect basal levels of Glil in $\mathrm{Smo}^{-/-}$cells, it may control the Hh signaling downstream of Ptch1. Interestingly, although the promoters of several Hh pathway components have putative consensus sequences for VDR binding ${ }^{93}$ the inhibitory effect on $\mathrm{Hh}$ seems to be independent of VDR binding by vitamin D3, because in $\mathrm{VDR}^{-/-}$cells the administration of calcitriol continues to repress Gli1 levels. ${ }^{98}$ Similar results have been also obtained using murine BCC cell lines. ${ }^{95}$

These data are in apparent contrast with what has been shown when using skin explants from VDR null mice, which did not present a reduction in Hh signaling following culture in the presence of vitamin D3, ${ }^{96}$ arguing in favor of a VDR requirement. One hypothesis to reconcile these different results is that vitamin D3 may exert both genomic (VDR dependent) and nongenomic (a direct action on Smo) ${ }^{94}$ effects, which may prevail alternatively in different cellular and physiological settings.

To evaluate the effectiveness of topical vitamin D3 treatment in BCC patients following the important preclinical data, a Phase III clinical trial has been initiated (NCT01358045) to clarify the feasibility and side effects of such treatments. In fact, one of the reasons why such treatment has not been extensively tested so far in different tumor types is because vitamin D3 through VDR acts on numerous physiological systems and its activation can lead to negative side effects. To facilitate clinical application, avoiding the side effects that cause prolonged administration of vitamin D3, researchers have recently produced semisynthetic derivatives that retain the ability to repress $\mathrm{Hh}$ and do not bind to VDR, which may be tested in future preclinical and clinical trials. ${ }^{99}$

\section{Blocking the Hh pathway upstream of Smo}

\section{Hh ligand antagonists}

A few years ago, a search for small molecules acting at the level of the Hh ligands was performed, identifying robotnikinin, a macrocyclic $\mathrm{Hh}$ inhibitor that is able to bind the extracellular Shh protein and block Shh signaling. ${ }^{100,101}$

More recently, starting from the structure of robotnikinin, several new macrocyclic analogs with increased $\mathrm{Hh}$ inhibitory activity have been generated. Interestingly, 
the most promising molecule (BRD-6851), in contrast to robotnikinin, is again a Smo antagonist. ${ }^{102}$

Another approach used to block the Hh pathway has been the generation of antibodies capable of binding Shh and to abolish its interaction with the Ptch receptor. The most effective and widely used antibody so far developed is called 5E1, which targets an epitope close to the region of Ptch/Shh interactions. ${ }^{103,104}$ This antibody has not so far been entered into any clinical trial.

\section{Oxysterol modulation}

Given the potential role of oxysterols in regulating the $\mathrm{Hh}$ pathway and more interestingly the fact that at least some oxysterols seem to be able to bind Smo on a site different to that which cyclopamine or Sag (Smo agonist) bind, ${ }^{36}$ they represent a potential alternative target. It is currently unclear how the oxysterols involved in Hh signaling regulation are synthesized, transported, and degraded such that their levels can be perturbed, but regulation of signaling sterol turnover might be another potential control point of the $\mathrm{Hh}$ pathway.

\section{Blocking the Hh pathway downstream of Smo}

\section{Hh pathway inhibitors (HPI)}

Hyman et al, in a large scale high-throughput screening for compounds that can inhibit Hh target gene expression induced by Smo and/or Sag, identified four molecules (HPI1-4) able to suppress $\mathrm{Hh}$ activity. ${ }^{105}$ They all act downstream of $\mathrm{SuFu}$, so they are not able to directly bind Smo, but they have a different mechanism of action: HPI1 acts on Gli1 (both exogenous and endogenous), HPI2 and HPI3 act on the ciliary process required for Gli2 function, and HPI4 interferes with ciliogenesis. The capacity of these inhibitors to act downstream of Smo is very promising in overcoming the resistance against Smo inhibitors. For this reason, Chenna et al generated a polymer nanoparticle-encapsulated formulation of HPI1 (NanoHHI) that is able to arrest proliferation in cells which ectopically express the Smo mutant resistant to vismodegib (D473H). ${ }^{106}$ Furthermore, NanoHHI reduced growth in a medulloblastoma xenograft model and, in combination with gemcitabine, significantly impeded growth in a pancreatic cancer xenograft and in orthotopic experiments with hepatocellular carcinoma cells. ${ }^{106,107}$

\section{Gli antagonists (GANT)}

Almost all the inhibitors of the Hh pathway that have been developed so far inhibit Smo activity. However, the development of molecules able to act further downstream would allow a direct hit on the final effectors of the signaling pathway (ie, Gli factors) and would provide a valid alternative in case of drug resistance.

Unfortunately, although searches for molecules acting on Gli factors have been performed for some years, the successful evidence provided is still limited to preclinical studies.

Lauth et al in 2007 identified two GANTs (GANT58 and GANT61) capable of inhibiting the transcriptional activity of Gli1 and Gli2: GANT61 is a hexahydropyrimidine while GANT58 has a thiophene core with four pyridine rings. ${ }^{77}$ Despite these structural differences, they both inhibit Gli1 and Gli2. The two drugs showed a strong inhibitory activity on pancreatic cancer cells and have worked mainly in reducing tumor growth in a xenograft model. ${ }^{77}$

Screens of 1990 synthetic chemicals and 94 natural products have identified a few compounds that can antagonize Hh target gene expression induced by Gli1 or Gli2 overexpression, including GANT58, GANT61, zerumbone, arcyriaflavin $\mathrm{C}$, and physalin. ${ }^{105}$ How these compounds antagonize Gli function has not been determined, although GANT61 appears to attenuate the DNA-binding activity of Gli1 in vivo and it has been suggested that arcyriaflavin $C$ and physalin $\mathrm{F}$ indirectly antagonize Gli function through protein kinase $\mathrm{C} /$ mitogen-activated protein kinase pathway blockade. Similarly, the natural product forskolin can nonselectively inhibit Hh signaling by activating adenylate cyclase and consequently protein kinase A.

The study of these inhibitors has been limited to preclinical models, and clinical trials are not currently under way. Recently it was shown that GANT61 causes apoptosis in myeloid leukemia cells and is able to synergize with rapamycin, ${ }^{108}$ and this raises hopes for further development of this type of antitumor drug.

\section{Regulation of Gli acetylation}

Another potential approach could be the use of negative regulators of histone deacetylase, which would maintain Gli acetylation and inhibit its transcriptional activity, as demonstrated by pharmacological inhibition with trichostatin A or overexpression of the $K C A S H$ family of genes, which drive histone deacetylase ubiquitination, targeting it for proteasomal degradation. ${ }^{37,109}$

\section{Retinoids: anticancer drugs with a hint of the Hh pathway?}

Retinoids have an important physiological role in the regulation of phenomena such as differentiation and 
proliferation, both during development and in adult life..$^{110-112}$ Experimental evidence exists that retinoids have antitumoral action, as in the case of acute promyelocytic leukemia, neuroblastoma, and other diseases. ${ }^{113,114}$

Several retinoids such as acitretin (NCT00020956) (15,116 $^{11}$ and tretinoin (NCT00007631) ${ }^{117}$ or tazarotene (see below) have been tested on $\mathrm{BCC}$, either in the form of topical cream or systemic therapies and both as treatment and chemopreventive therapies. Tazarotene was the first retinoid to arouse some interest as a potential drug for the treatment of BCC. ${ }^{118-120}$ In clinical settings, tazarotene use is approved for the treatment of psoriasis and acne and is well tolerated in patients undergoing an oral or topical regimen. ${ }^{119,121}$

In 2004, it was demonstrated (on Ptch $1^{+/-}$mice exposed to ultraviolet or ionizing radiation) that tazarotene inhibited the formation of new microscopic lesions and reduced BCC size. ${ }^{122}$ In the same year, Bianchi et al presented positive results of an experimental administration of tazarotene locally to BCC patients (gel $0.1 \%$ ) once a day for 24 weeks. At the end of the study, $70 \%$ of lesions showed response to treatment and about $30 \%$ healed with no disease recurrence in 3 years of follow-up. ${ }^{119}$

There are currently two Phase II clinical trials in patients with BCNS (NCT00783965; NCT00489086) with the purpose of evaluating the preventive effect of this highly tolerable topical drug on BCC formation.

The antitumor effects of retinoids are elicited mainly by inducing apoptosis and differentiation, although the mechanisms have not been fully assessed. It is not clear if and how tazarotene or other retinoids may act on the Hh pathway, although a link has been suggested by Goyette et al. ${ }^{123}$

Whether $\mathrm{Hh}$ and retinoids crosstalk or not needs to be further verified, but combined therapies with $\mathrm{Hh}$ inhibitors may provide a useful and promising tool, given their relative tolerability.

\section{Overcoming resistance to $\mathrm{Hh} / \mathrm{Smo}$ inhibitors: phosphatidylinositol 3-kinase and epidermal growth factor receptor inhibition}

Experimental evidence points to the potential acquisition of tumor resistance to Smo antagonist treatment, both by Smo receptor mutations or by amplification of Hh pathway downstream target genes such as CCND1 and Gli2. ${ }^{66,85,124}$

Recently a few studies have shown the possibility of overcoming drug resistance through the use of inhibitors of other pathways, which are able to interplay or act in synergy with Hh to promote tumorigenesis or tumor proliferation:

- Researchers at Novartis have shown that mechanisms of LDE225 resistance can be overcome by the use of inhibitors of phosphatidylinositol 3-kinase. ${ }^{66}$ Importantly, this information has been confirmed by another group on vismodegib-resistant tumors. ${ }^{85}$

- Another pathway which may cooperate with Hh in BCC is epidermal growth factor receptor signaling. ${ }^{125,126}$ Recent data in vitro in human cell lines and in vivo in mouse models suggest the relevance of targeting this cooperation as a new therapeutic hypothesis to be further developed. ${ }^{126}$

\section{Conclusion and future directions}

The identification of the $\mathrm{Hh}$ pathway as a strategic target for BCC treatment has prompted the search of Hh inhibitors for therapeutic purposes. Relatively quickly a first molecule has been recently approved for clinical use by the FDA, and other molecules are undergoing clinical trials. Several drugs already approved for other therapies have also been tested and proven to be capable of suppressing Hh activity (Figure 1). At the moment, most of the molecules that have shown therapeutic efficacy target the Smo intramembrane receptor, either in its function or in its capacity to migrate into the cilium, indicating Smo as a critical component of the pathway. Unfortunately, the possibility of BCC developing resistance to Smo-targeting drugs (and in general to drugs targeting a single structure) is evident, and there have already been published papers documenting both mutations of the receptor or constitutive activation of the Hh pathway downstream of Smo. For this reason, other drugs which target other structures of the Smo receptor, or even better with different downstream targets in the Hh pathway (including GANTs), need to be developed.

One of the reasons for which it is so strategic to suppress the Hh pathway in BCC is not only the specific role of $\mathrm{Hh}$ on initiation and promotion in this type of tumor, but also the increasing evidence of the role of the Hh pathway in the maintenance of the stem cell and cancer stem cell compartments. ${ }^{127,128}$ Targeting Hh could reduce the pool of cancer stem cells remaining after chemotherapy and in this way reduce tumor recurrence.

Furthermore, since Hh is not the only mechanism involved in tumor formation and maintenance, the use of combinatorial treatment is recommended to increase efficiency of the cure.

Another issue to be dealt with is the molecular characterization of the patient's BCC, to avoid unnecessary 
delay in the identification of the best therapeutic approach (ie, do not use target Smo if Smo is mutated). Molecular characterization may be very expensive and in order to reduce the costs it is also necessary to work on the identification of a few discriminating target molecules to be analyzed.

In summary, the approval of vismodegib has provided a new and important tool for BCC treatment, but further efforts will be needed to find alternative drugs for resistant tumors and may be a more economical solution (considering the potential cost of vismodegib is currently about USD7500 per month and the world market is evaluated at at least USD1-4 billion per year). ${ }^{56}$ Although competition, which was strong until FDA's approval of vismodegib, may fade in the future, a substantial number of clinical trials are currently ongoing and there is a good chance that other useful therapeutic instruments will be available shortly.

\section{Acknowledgments}

This work was supported, in part, by Associazione Italiana Ricerca Cancro MIUR (FIRB and PRIN projects), Ministry of Health, Fondazione Roma, Fondazione Mariani, EU Healing grant, Italian Institute of Technology, and Agenzia Spaziale Italiana.

\section{Disclosure}

The authors report no conflicts of interest in this work.

\section{References}

1. American Cancer Society. Cancer Facts and Figures 2012. Atlanta, GA: American Cancer Society; 2012. Available from: http://www.cancer.org/ acs/groups/content/@epidemiologysurveilance/documents/document/ acspc-031941.pdf. Accessed November 5, 2012.

2. Kim RH, Armstrong AW. Nonmelanoma skin cancer. Dermatol Clin. 2012;30(1):125-139.

3. Rubin AI, Chen EH, Ratner D. Basal-cell carcinoma. N Engl J Med. 2005;353(21):2262-2269.

4. Kasper M, Jaks V, Hohl D, Toftgard R. Basal cell carcinoma-molecular biology and potential new therapies. J Clin Invest. 2012;122(2): 455-463.

5. Karagas MR, Weinstock MA, Nelson HH. Keratinocyte carcinomas (basal and squamous cell carcinomas of the skin). In: Schottenfeld D, Fraumeni JF Jr, editors. Cancer Epidemiology and Prevention, 3rd ed. Oxford: Oxford University Press; 2006:1230-1250.

6. Weiss GJ, Korn RL. Metastatic basal cell carcinoma in the era of Hedgehog signaling pathway inhibitors. Cancer. 2012;118(21):5310-5319.

7. Walling HW, Fosko SW, Geraminejad PA, Whitaker DC, Arpey CJ. Aggressive basal cell carcinoma: presentation, pathogenesis, and management. Cancer Metastasis Rev. 2004;23(3-4):389-402.

8. Lomas A, Leonardi-Bee J, Bath-Hextall F. A systematic review of worldwide incidence of nonmelanoma skin cancer. $\mathrm{Br} J$ Dermatol. 2012;166(5):1069-1080.

9. Guy GP, Ekwueme DU. Years of potential life lost and indirect costs of melanoma and non-melanoma skin cancer: a systematic review of the literature. Pharmacoeconomics. 2011;29(10):863-874.

10. Weinstock MA, Still JM. Assessing current treatment options for patients with severe/advanced basal cell carcinoma. Semin Cutan Med Surg. 2011;30(Suppl 4):S10-S13.
11. Marcil I, Stern RS. Risk of developing a subsequent nonmelanoma skin cancer in patients with a history of nonmelanoma skin cancer: a critical review of the literature and meta-analysis. Arch Dermatol. 2000;136(12):1524-1530.

12. Desai T, Chen CL, Desai A, Kirby W. Basic pharmacology of topical imiquimod, 5-fluorouracil, and diclofenac for the dermatologic surgeon. Dermatol Surg. 2012;38(1):97-103.

13. Lee S, Selva D, Huilgol SC, Goldberg RA, Leibovitch I. Pharmacological treatments for basal cell carcinoma. Drugs. 2007;67(6):915-934.

14. Teglund S, Toftgard R. Hedgehog beyond medulloblastoma and basal cell carcinoma. Biochim Biophys Acta. 2010;1805(2):181-208.

15. Ingham PW, Nakano Y, Seger C. Mechanisms and functions of Hedgehog signalling across the metazoa. Nat Rev Genet. 2011;12(6):393-406.

16. Caro I, Low JA. The role of the Hedgehog signaling pathway in the development of basal cell carcinoma and opportunities for treatment. Clin Cancer Res. 2010;16(13):3335-3339.

17. Ng JM, Curran T. The Hedgehog's tale: developing strategies for targeting cancer. Nat Rev Cancer. 2011;11(7):493-501.

18. Po A, Ferretti E, Miele E, et al. Hedgehog controls neural stem cells through p53-independent regulation of Nanog. EMBO J. 2010;29(15): 2646-2658.

19. Takebe N, Harris PJ, Warren RQ, Ivy SP. Targeting cancer stem cells by inhibiting Wnt, Notch, and Hedgehog pathways. Nat Rev Clin Oncol. 2011;8(2):97-106.

20. Varjosalo M, Taipale J. Hedgehog signaling. J Cell Sci. 2007; 120(Pt 1):3-6.

21. Ingham PW, McMahon AP. Hedgehog signaling in animal development: paradigms and principles. Genes Dev. 2001;15(23):3059-3087.

22. Barakat MT, Humke EW, Scott MP. Learning from Jekyll to control Hyde: Hedgehog signaling in development and cancer. Trends $\mathrm{Mol}$ Med. 2010;16(8):337-348.

23. Lum L, Beachy PA. The Hedgehog response network: sensors, switches, and routers. Science. 2004;304(5678):1755-1759.

24. Hooper JE, Scott MP. Communicating with Hedgehogs. Nat Rev Mol Cell Biol. 2005;6(4):306-317.

25. Ingham PW, Placzek M. Orchestrating ontogenesis: variations on a theme by Sonic Hedgehog. Nat Rev Genet. 2006;7(11):841-850.

26. Towers M, Tickle C. Growing models of vertebrate limb development. Development. 2009;136(2):179-190.

27. Mann RK, Beachy PA. Novel lipid modifications of secreted protein signals. Annu Rev Biochem. 2004;73:891-923.

28. Therond PP. Release and transportation of Hedgehog molecules. Curr Opin Cell Biol. 2012;24(2):173-180.

29. Rohatgi R, Milenkovic L, Scott MP. Patched1 regulates Hedgehog signaling at the primary cilium. Science. 2007;317(5836):372-376.

30. Wollam J, Antebi A. Sterol regulation of metabolism, homeostasis, and development. Annu Rev Biochem. 2011;80:885-916.

31. Corcoran RB, Scott MP. Oxysterols stimulate Sonic Hedgehog signal transduction and proliferation of medulloblastoma cells. Proc Natl Acad Sci US A. 2006;103(22):8408-8413.

32. Strutt H, Thomas C, Nakano Y, et al. Mutations in the sterol-sensing domain of Patched suggest a role for vesicular trafficking in Smoothened regulation. Curr Biol. 2001;11(8):608-613.

33. Dwyer JR, Sever N, Carlson M, Nelson SF, Beachy PA, Parhami F. Oxysterols are novel activators of the Hedgehog signaling pathway in pluripotent mesenchymal cells. J Biol Chem. 2007;282(12):8959-8968.

34. Johnson JS, Meliton V, Kim WK, et al. Novel oxysterols have pro-osteogenic and anti-adipogenic effects in vitro and induce spinal fusion in vivo. J Cell Biochem. 2011;112(6):1673-1684.

35. Sharpe HJ, de Sauvage FJ. Signaling: an oxysterol ligand for Smoothened. Nat Chem Biol. 2012;8(2):139-140.

36. Nachtergaele S, Mydock LK, Krishnan K, et al. Oxysterols are allosteric activators of the oncoprotein Smoothened. Nat Chem Biol. 2012;8(2):211-220.

37. Canettieri G, Di Marcotullio L, Greco A, et al. Histone deacetylase and Cullin3-REN(KCTD11) ubiquitin ligase interplay regulates Hedgehog signalling through Gli acetylation. Nat Cell Biol. 2010;12(2): $132-142$. 
38. Pasca di Magliano M, Hebrok M. Hedgehog signalling in cancer formation and maintenance. Nat Rev Cancer. 2003;3(12):903-911.

39. Gorlin RJ. Nevoid basal-cell carcinoma syndrome. Medicine (Baltimore). 1987;66(2):98-113.

40. Hahn H, Wicking C, Zaphiropoulous PG, et al. Mutations of the human homolog of Drosophila patched in the nevoid basal cell carcinoma syndrome. Cell. 1996;85(6):841-851.

41. Johnson RL, Rothman AL, Xie J, et al. Human homolog of patched, a candidate gene for the basal cell nevus syndrome. Science. 1996;272(5268):1668-1671

42. Aszterbaum M, Rothman A, Johnson RL, et al. Identification of mutations in the human PATCHED gene in sporadic basal cell carcinomas and in patients with the basal cell nevus syndrome. $J$ Invest Dermatol. 1998;110(6):885-888.

43. Reifenberger J, Wolter M, Knobbe CB, et al. Somatic mutations in the PTCH, SMOH, SUFUH and TP53 genes in sporadic basal cell carcinomas. Br J Dermatol. 2005;152(1):43-51.

44. Wolter M, Reifenberger J, Sommer C, et al. Mutations in the human homologue of the Drosophila segment polarity gene patched (PTCH) in sporadic basal cell carcinomas of the skin and primitive neuroectodermal tumors of the central nervous system. Cancer Res. 1997;57(13):2581-2585.

45. Lam CW, Xie J, To KF, et al. A frequent activated smoothened mutation in sporadic basal cell carcinomas. Oncogene. 1999;18(3):833-836.

46. Xie J, Murone M, Luoh SM, et al. Activating Smoothened mutations in sporadic basal-cell carcinoma. Nature. 1998;391(6662):90-92.

47. Reifenberger J, Wolter M, Weber RG, et al. Missense mutations in $\mathrm{SMOH}$ in sporadic basal cell carcinomas of the skin and primitive neuroectodermal tumors of the central nervous system. Cancer Res. 1998;58(9):1798-1803.

48. Oro AE, Higgins KM, Hu Z, Bonifas JM, Epstein EH Jr, Scott MP. Basal cell carcinomas in mice overexpressing Sonic Hedgehog. Science. 1997;276(5313):817-821.

49. Keeler RF. Teratogenic compounds of Veratrum californicum (Durand) X. Cyclopia in rabbits produced by cyclopamine. Teratology. 1970;3(2): 175-180.

50. Incardona JP, Gaffield W, Kapur RP, Roelink H. The teratogenic Veratrum alkaloid cyclopamine inhibits Sonic Hedgehog signal transduction. Development. 1998;125(18):3553-3562.

51. Taipale J, Chen JK, Cooper MK, et al. Effects of oncogenic mutations in Smoothened and Patched can be reversed by cyclopamine. Nature. 2000;406(6799):1005-1009.

52. Dahmane N, Sanchez P, Gitton Y, et al. The Sonic Hedgehog-Gli pathway regulates dorsal brain growth and tumorigenesis. Development. 2001;128(24):5201-5212.

53. Chen JK, Taipale J, Cooper MK, Beachy PA. Inhibition of Hedgehog signaling by direct binding of cyclopamine to Smoothened. Genes Dev. 2002;16(21):2743-2748

54. Tabs S, Avci O. Induction of the differentiation and apoptosis of tumor cells in vivo with efficiency and selectivity. Eur J Dermatol. 2004;14(2): 96-102.

55. De Smaele E, Ferretti E, Gulino A. Vismodegib, a small-molecule inhibitor of the Hedgehog pathway for the treatment of advanced cancers. Curr Opin Investig Drugs. 2010;11(6):707-718.

56. Dlugosz A, Agrawal S, Kirkpatrick P. Vismodegib. Nat Rev Drug Discov. 2012;11(6):437-438.

57. Robarge KD, Brunton SA, Castanedo GM, et al. GDC-0449 - a potent inhibitor of the Hedgehog pathway. Bioorg Med Chem Lett. 2009;19(19):5576-5581.

58. Von Hoff DD, LoRusso PM, Rudin CM, et al. Inhibition of the Hedgehog pathway in advanced basal-cell carcinoma. $N$ Engl $\mathrm{J}$ Med. 2009;361(12):1164-1172.

59. Sekulic A, Migden MR, Oro AE, et al. Efficacy and safety of vismodegib in advanced basal-cell carcinoma. N Engl J Med. 2012;366(23) 2171-2179.

60. US Food and Drug Administration. FDA labeling information: Erivedge. 2012. Available from: http://www.accessdata.fda.gov/drugsatfda_docs/ label/2012/203388 lbl.pdf. Accessed November 5, 2012.
61. Tang JY, Mackay-Wiggan JM, Aszterbaum M, et al. Inhibiting the Hedgehog pathway in patients with the basal-cell nevus syndrome. N Engl J Med. 2012;366(23):2180-2188.

62. Rudin CM, Hann CL, Laterra J, et al. Treatment of medulloblastoma with Hedgehog pathway inhibitor GDC-0449. $N$ Engl $J$ Med. 2009;361(12):1173-1178.

63. Yauch RL, Dijkgraaf GJ, Alicke B, et al. Smoothened mutation confers resistance to a Hedgehog pathway inhibitor in medulloblastoma. Science. 2009;326(5952):572-574.

64. Williams JA, Guicherit OM, Zaharian BI, et al. Identification of a small molecule inhibitor of the Hedgehog signaling pathway: effects on basal cell carcinoma-like lesions. Proc Natl Acad Sci U S A. 2003;100(8): 4616-4621.

65. Frank-Kamenetsky M, Zhang XM, Bottega S, et al. Small-molecule modulators of Hedgehog signaling: identification and characterization of Smoothened agonists and antagonists. $J$ Biol. 2002;1(2):10.

66. Buonamici S, Williams J, Morrissey M, et al. Interfering with resistance to smoothened antagonists by inhibition of the PI3K pathway in medulloblastoma. Sci Transl Med. 2010;2(51):51ra70.

67. Skvara H, Kalthoff F, Meingassner JG, et al. Topical treatment of basal cell carcinomas in nevoid basal cell carcinoma syndrome with a smoothened inhibitor. J Invest Dermatol. 2011;131(8): $1735-1744$.

68. Novartis. Innovation Updates: Key Developments in the Fourth Quarter of 2011. Basel: Novartis; 2012. Available from: http://www.novartis. com/downloads/investors/financial-results/q4-2011-innovation-tables. pdf. Accessed November 5, 2012.

69. Park KS, Martelotto LG, Peifer M, et al. A crucial requirement for Hedgehog signaling in small cell lung cancer. Nat Med. 2011;17(11): 1504-1508.

70. Tang JY, Marghoob AA. Emerging treatments and signaling pathway inhibitors. Semin Cutan Med Surg. 2011;30(Suppl 4): S14-S18.

71. Lappano R, Maggiolini M. G protein-coupled receptors: novel targets for drug discovery in cancer. Nat Rev Drug Discov. 2011;10(1): $47-60$.

72. Tremblay MR, Nesler M, Weatherhead R, Castro AC. Recent patents for Hedgehog pathway inhibitors for the treatment of malignancy. Expert Opin Ther Pat. 2009;19(8):1039-1056.

73. Tremblay MR, Lescarbeau A, Grogan MJ, et al. Discovery of a potent and orally active Hedgehog pathway antagonist (IPI-926). J Med Chem. 2009;52(14):4400-4418.

74. Tremblay MR, McGovern K, Read MA, Castro AC. New developments in the discovery of small molecule Hedgehog pathway antagonists. Curr Opin Chem Biol. 2010;14(3):428-435.

75. Lee MJ, Hatton BA, Villavicencio EH, et al. Hedgehog pathway inhibitor saridegib (IPI-926) increases lifespan in a mouse medulloblastoma model. Proc Natl Acad Sci U S A. 2012;109(20):7859-7864.

76. Dean M, Rzhetsky A, Allikmets R. The human ATP-binding cassette (ABC) transporter superfamily. Genome Res. 2001;11(7): $1156-1166$

77. Lauth M, Bergstrom A, Shimokawa T, Toftgard R. Inhibition of GLI-mediated transcription and tumor cell growth by small-molecule antagonists. Proc Natl Acad Sci U S A. 2007;104(20):8455-8460.

78. Robey RW, Massey PR, Amiri-Kordestani L, Bates SE. ABC transporters: unvalidated therapeutic targets in cancer and the CNS. Anticancer Agents Med Chem. 2010;10(8):625-633.

79. Zhang Y, Laterra J, Pomper MG. Hedgehog pathway inhibitor HhAntag691 is a potent inhibitor of ABCG2/BCRP and ABCB1/Pgp. Neoplasia. 2009;11(1):96-101.

80. Wong H, Chen JZ, Chou B, et al. Preclinical assessment of the absorption, distribution, metabolism and excretion of GDC-0449 (2-chloro-N-(4-chloro-3-(pyridin-2-yl)phenyl)-4-(methylsulfonyl) benzamide), an orally bioavailable systemic Hedgehog signalling pathway inhibitor. Xenobiotica. 2009;39(11):850-861.

81. Rudin CM, Weiss GJ, Chang A, et al. A phase 1 study of IPI-926, an inhibitor of the Hedgehog pathway, in patients with advanced or metastatic solid tumors [abstract]. Ann Oncol. 2010;21(Suppl 8):viii164. 
82. Munchhof MJ, Li Q, Shavnya A, et al. Discovery of PF-04449913, a potent and orally bioavailable inhibitor of Smoothened. ACS Med Chem Lett. 2012;3(2):106-111.

83. Jamieson C, Cortes JE, Oehler V, et al. Phase 1 dose-escalation study of PF-04449913, an oral Hedgehog (Hh) inhibitor, in patients with select hematologic malignancies [abstract]. Blood. 2011;118:424.

84. Papayannidis C, Guadagnuolo V, Iacobucci I, et al. PF-04449913 reverts multi drug resistance (MDR) by a strong down-regulation of ABCA2 and BCL2 on leukemia stem cells in phase I acute myeloid leukemia and chronic myeloid leukemia treated patients [abstract]. Cancer Res. 2012;72(8 Suppl 1):4619.

85. Dijkgraaf GJ, Alicke B, Weinmann L, et al. Small molecule inhibition of GDC-0449 refractory smoothened mutants and downstream mechanisms of drug resistance. Cancer Res. 2011;71(2): 435-444.

86. Yang B, Hird AW, Russell DJ, et al. Discovery of novel Hedgehog antagonists from cell-based screening: isosteric modification of p38 bisamides as potent inhibitors of SMO. Bioorg Med Chem Lett. 2012;22(14):4907-4911.

87. Kim J, Tang JY, Gong R, et al. Itraconazole, a commonly used antifungal that inhibits Hedgehog pathway activity and cancer growth. Cancer Cell. 2010;17(4):388-399.

88. Kim D, Kim J, Spaunhurst K, et al. An open-label, exploratory phase II study of oral itraconazole for the treatment of basal cell carcinoma [abstract]. Cancer Res. 2012;72(Suppl 8):LB-223.

89. Nacev BA, Grassi P, Dell A, Haslam SM, Liu JO. The antifungal drug itraconazole inhibits vascular endothelial growth factor receptor 2 (VEGFR2) glycosylation, trafficking, and signaling in endothelial cells. J Biol Chem. 2011;286(51):44045-44056.

90. Shi W, Nacev BA, Aftab BT, Head S, Rudin CM, Liu JO. Itraconazole side chain analogues: structure-activity relationship studies for inhibition of endothelial cell proliferation, vascular endothelial growth factor receptor 2 (VEGFR2) glycosylation, and Hedgehog signaling. J Med Chem. 2011;54(20):7363-7374

91. Brunton SA, Stibbard JH, Rubin LL, et al. Potent inhibitors of the Hedgehog signaling pathway. J Med Chem. 2008;51(5): 1108-1110.

92. Deeb KK, Trump DL, Johnson CS. Vitamin D signalling pathways in cancer: potential for anticancer therapeutics. Nat Rev Cancer. 2007;7(9):684-700.

93. Bikle DD. Vitamin D and the skin: physiology and pathophysiology. Rev Endocr Metab Disord. 2012;13(1):3-19.

94. Bijlsma MF, Spek CA, Zivkovic D, van de Water S, Rezaee F, Peppelenbosch MP. Repression of smoothened by patched-dependent (pro-)vitamin D3 secretion. PLoS Biol. 2006;4(8):e232.

95. Tang JY, Xiao TZ, Oda Y, et al. Vitamin D3 inhibits Hedgehog signaling and proliferation in murine basal cell carcinomas. Cancer Prev Res (Phila). 2011;4(5):744-751.

96. Teichert AE, Elalieh H, Elias PM, Welsh J, Bikle DD. Overexpression of Hedgehog signaling is associated with epidermal tumor formation in vitamin D receptor-null mice. J Invest Dermatol. 2011;131(11):2289-2297.

97. Kamradt J, Rafi L, Mitschele T, et al. Analysis of the vitamin D system in cutaneous malignancies. Recent Results Cancer Res. 2003;164:259-269.

98. Uhmann A, Niemann H, Lammering B, et al. Antitumoral effects of calcitriol in basal cell carcinomas involve inhibition of Hedgehog signaling and induction of vitamin D receptor signaling and differentiation. Mol Cancer Ther. 2011;10(11):2179-2188.

99. Deberardinis AM, Banerjee U, Miller M, Lemieux S, Hadden MK. Probing the structural requirements for vitamin D3 inhibition of the Hedgehog signaling pathway. Bioorg Med Chem Lett. 2012; 22(14):4859-4863.

100. Stanton BZ, Peng LF, Maloof N, et al. A small molecule that binds Hedgehog and blocks its signaling in human cells. Nat Chem Biol. 2009;5(3):154-156.
101. Peng LF, Stanton BZ, Maloof N, Wang X, Schreiber SL. Syntheses of aminoalcohol-derived macrocycles leading to a small-molecule binder to and inhibitor of Sonic Hedgehog. Bioorg Med Chem Lett. 2009;19:6319-6325.

102. Dockendorff C, Nagiec MM, Weiwer M, et al. Macrocyclic Hedgehog pathway inhibitors: optimization of cellular activity and mode of action studies. ACS Med Chem Lett. 2012;3(10):808-813.

103. Ericson J, Morton S, Kawakami A, Roelink H, Jessell TM. Two critical periods of Sonic Hedgehog signaling required for the specification of motor neuron identity. Cell. 1996;87(4):661-673.

104. Pepinsky RB, Rayhorn P, Day ES, et al. Mapping Sonic Hedgehog-receptor interactions by steric interference. J Biol Chem. 2000;275(15):10995-11001.

105. Hyman JM, Firestone AJ, Heine VM, et al. Small-molecule inhibitors reveal multiple strategies for Hedgehog pathway blockade. Proc Natl Acad Sci US A. 2009;106(33):14132-14137.

106. Chenna V, Hu C, Pramanik D, et al. A polymeric nanoparticle encapsulated small-molecule inhibitor of Hedgehog signaling (NanoHHI) bypasses secondary mutational resistance to Smoothened antagonists. Mol Cancer Ther. 2012;11(1):165-173.

107. Xu Y, Chenna V, Hu C, et al. Polymeric nanoparticle-encapsulated Hedgehog pathway inhibitor HPI-1 (NanoHHI) inhibits systemic metastases in an orthotopic model of human hepatocellular carcinoma. Clin Cancer Res. 2012;18(5):1291-1302.

108. Pan D, Li Y, Li Z, Wang Y, Wang P, Liang Y. Gli inhibitor GANT61 causes apoptosis in myeloid leukemia cells and acts in synergy with rapamycin. Leuk Res. 2012;36(6):742-748.

109. De Smaele E, Di Marcotullio L, Moretti M, et al. Identification and characterization of $\mathrm{KCASH} 2$ and $\mathrm{KCASH} 3,2$ novel Cullin3 adaptors suppressing histone deacetylase and Hedgehog activity in medulloblastoma. Neoplasia. 2011;13(4):374-385.

110. Gutierrez-Mazariegos J, Theodosiou M, Campo-Paysaa F, Schubert M. Vitamin A: a multifunctional tool for development. Semin Cell Dev Biol. 2011;22(6):603-610.

111. Gudas LJ. Emerging roles for retinoids in regeneration and differentiation in normal and disease states. Biochim Biophys Acta. 2012;1821(1):213-221.

112. Tang XH, Gudas LJ. Retinoids, retinoic acid receptors, and cancer. Annu Rev Pathol. 2011;6:345-364.

113. Hansen LA, Sigman CC, Andreola F, Ross SA, Kelloff GJ, De Luca LM. Retinoids in chemoprevention and differentiation therapy. Carcinogenesis. 2000;21(7):1271-1279.

114. Altucci L, Leibowitz MD, Ogilvie KM, de Lara AR, Gronemeyer H. RAR and RXR modulation in cancer and metabolic disease. Nat Rev Drug Discov. 2007;6(10):793-810.

115. Ingves $\mathrm{C}$, Jemec GB. Combined imiquimod and acitretin for nonsurgical treatment of basal cell carcinoma. Scand J Plast Reconstr Surg Hand Surg. 2003;37(5):293-295.

116. Lien MH, Fenske NA, Glass LF. Advances in the chemoprevention of non-melanoma skin cancer in high-risk organ transplant recipients. Semin Oncol. 2012;39(2):134-138.

117. Weinstock MA, Bingham SF, Digiovanna JJ, et al. Tretinoin and the prevention of keratinocyte carcinoma (basal and squamous cell carcinoma of the skin): a Veterans Affairs randomized chemoprevention trial. J Invest Dermatol. 2012;132(6):1583-1590.

118. Chandraratna RA. Tazarotene - first of a new generation of receptorselective retinoids. Br J Dermatol. 1996;135(Suppl 49):18-25.

119. Bianchi L, Orlandi A, Campione E, et al. Topical treatment of basal cell carcinoma with tazarotene: a clinicopathological study on a large series of cases. Br J Dermatol. 2004;151(1):148-156.

120. Orlandi A, Bianchi L, Costanzo A, Campione E, Giusto Spagnoli L, Chimenti S. Evidence of increased apoptosis and reduced proliferation in basal cell carcinomas treated with tazarotene. $J$ Invest Dermatol. 2004;122(4):1037-1041.

121. Jones PH, Burnett RD, Fainaru I, et al. A phase 1 study of tazarotene in adults with advanced cancer. Br J Cancer. 2003;89(5):808-815. 
122. So PL, Lee K, Hebert J, et al. Topical tazarotene chemoprevention reduces basal cell carcinoma number and size in Ptch1+/- mice exposed to ultraviolet or ionizing radiation. Cancer Res. 2004;64(13): 4385-4389.

123. Goyette P, Allan D, Peschard P, Chen CF, Wang W, Lohnes D. Regulation of Gli activity by all-trans retinoic acid in mouse keratinocytes. Cancer Res. 2000;60(19):5386-5389.

124. Metcalfe C, de Sauvage FJ. Hedgehog fights back: mechanisms of acquired resistance against Smoothened antagonists. Cancer Res. 2011;71(15):5057-5061.

125. Kasper M, Schnidar H, Neill GW, et al. Selective modulation of Hedgehog/GLI target gene expression by epidermal growth factor signaling in human keratinocytes. Mol Cell Biol. 2006;26(16): 6283-6298.
126. Eberl M, Klinger S, Mangelberger D, et al. Hedgehog-EGFR cooperation response genes determine the oncogenic phenotype of basal cell carcinoma and tumour-initiating pancreatic cancer cell. EMBO Mol Med. 2012;4(3):218-233.

127. Sellheyer K. Basal cell carcinoma: cell of origin, cancer stem cell hypothesis and stem cell markers. Br J Dermatol. 2011;164(4):696-711.

128. Merchant AA, Matsui W. Targeting Hedgehog - a cancer stem cell pathway. Clin Cancer Res. 2010;16(12):3130-3140.

\section{Publish your work in this journal}

The Journal of Experimental Pharmacology is an international, peerreviewed, open access journal publishing original research, reports, reviews and commentaries on all areas of laboratory and experimental pharmacology. The manuscript management system is completely online and includes a very quick and fair peer-review system.
Visit http://www.dovepress.com/testimonials.php to read real quotes from published authors. 